\title{
Animal Surveillance In NBC Defensive Operations
}

\section{Introduction}

Many Biological Agents (BA) used in Biological Warfare (BW) also cause disease in animal populations. The onset of signs and symptoms in man may therefore coincide with illness or death within animal populations.

Animal surveillance can be useful in three distinct areas of medical intelligence, namely:

- Enhancement of prior knowledge of zoonotic threats to human health in theatre.

- Detection of BW and CW incidents, and outbreaks of zoonotic disease.

- Collection of diagnostic and forensic evidence after a BW or CW incident.

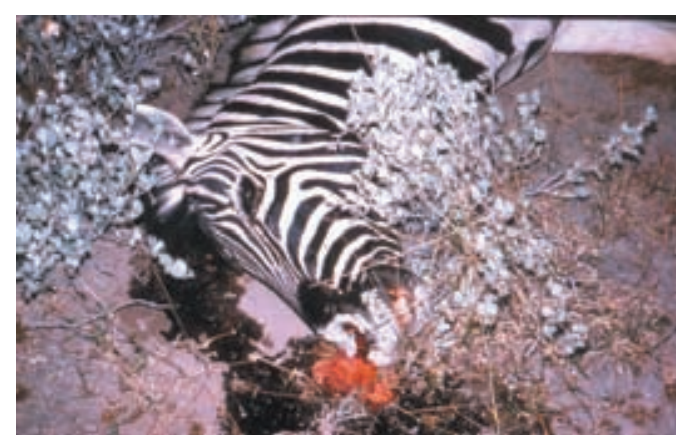

Fig 1. Anthrax in a herbivore.

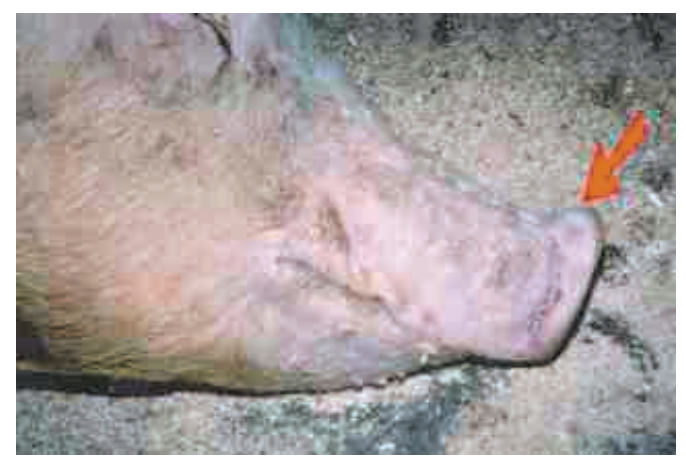

Maj TS Cottrell MSc BVMS MRCVS RAVC CBRN Operations, Directorate of Stratigic Support, Ministry of Defence, Winterbourne Gunner, Salisbury, Wilts, SP4 OES

Capt ER Morgan PhD MA Vet MB MRCVS RAVC (V)

University of Bristol, School of Biological Sciences, Woodland Road, Bristol, BS8 1UG following ways:

- Clinical signs may appear in animals before the human population where:

- A BA has a shorter incubation period in animals than in humans.

- Exposure of animals to the agent occurs before exposure of the human population.

- The infectious dose in animals is lower than in the humans.

In this event, loss of life would be minimised by maximising force protection through timely instigation of force protection measures such as the use of Medical Countermeasures (MedCM) or Restriction of Movement (ROM) in the human population at risk.

- The additional information provided by the identification of disease in animals may allow a definitive diagnosis to be reached appreciably earlier than using data from humans alone. The death of animals may provide an opportunity for post mortem examination and sampling, an option that may not be available within the human population. Where clinical disease is evident in animals, cases may be selected for euthanasia and early post mortem examination.

- The effects of toxins and chemical agents on most mammalian species are very similar to those on man, and the observation of clinical signs and collection of samples from animals sick or found dead may allow early definitive diagnosis in these cases also.

- The pattern of disease in animals may itself contribute to the identification of a BA, and help to distinguish it from endemic or coincidental disease in humans. Many diseases caused by BA present with rather vague symptoms in man, and concurrent outbreaks of disease in animals may alert medical services to the possibility of BW attack.

- The time-course and geographical distribution of disease in animals can be used to indicate affected areas, and provide some indication of the persistence of the threat following delivery of BA.

\section{WARNING AND DETECTION}

\section{Intelligence}

Adequate, accurate and timely intelligence is required in order to develop an effective defence against BW. Knowledge of 


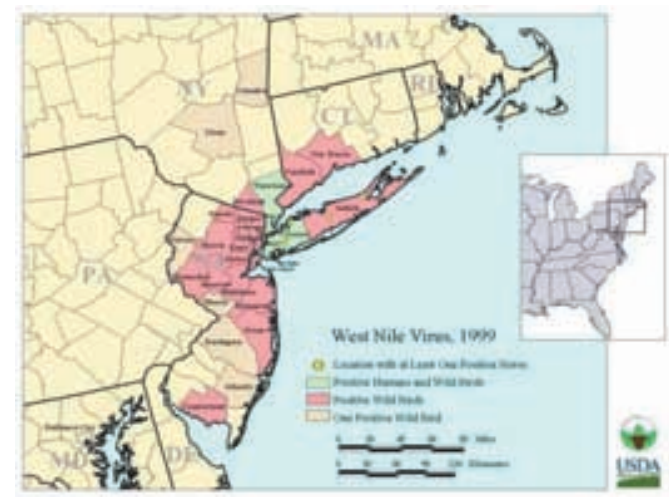

Fig 3. West Nile fever 1999. (countesy of CDC Atlanta).

operation-specific BW or CW threats, or detection of agents or casualties, will help to target animal surveillance efforts. However, since disease in animals may be the first sign of BW attack in particular, a broad minded approach to the diagnosis of animal disease is needed, including veterinary consideration of differential diagnoses beyond acknowledged BW threats or expected endemic diseases.

\section{Detection of BW Attack}

BA aerosol detectors can be configured to detect general hazards or specific agents. The positioning of detectors and their sensitivity are critical to their performance. They will be deployed as a network and may be described as located relative to personnel in two ways:

- Upwind of personnel in order to give prior warning of an attack before exposure occurs.

- Co-located with personnel to confirm that exposure has occurred, and aspirationally to provide a detect-to-warn capability.

It should be noted that a single detector may act in both these ways simultaneously for personnel in different locations.

\section{Epidemiological Monitoring}

After an undetected BA attack the first sign of an event may be the appearance of casualties (military, civilian; human or animal). The earlier patterns of an emerging outbreak of a disease are recognised, the greater is the chance of protecting troops who have not yet developed symptoms. Advanced medical surveillance and epidemiological reporting systems with collation and analysis of data can be used to identify spikes of disease on a real time basis. The background level of naturally occurring BW diseases is a critical information requirement for epidemiological reporting. These systems also play an important part in the subsequent assessment and management of a recognised attack. In this context, rapidly deployable biological medical reconnaissance of unusual outbreaks of diseases in the theatre is important.

Differential Diagnosis of BW Agents The Veterinary Officer (VO) must attempt to distinguish between an epidemic (epizootic) of natural origin and a BW attack. In practice, force protection measures may well be similar whether the risk of human infection is from BW or from a natural zoonotic outbreak. However, the psychological, political and intelligence implications of a BW attack dictate that suggestions of deliberate use must not be made lightly. Conditions in theatre may themselves cause outbreaks of zoonotic disease in the absence of BW, and some BW agents can be confused with endemic disease in animals, zoonotic or not. BA might also be released accidentally (Release Other Than Attack - ROTA). Diagnosis of potential zoonoses should also be made carefully, in order to avoid unnecessary counter-measures, diversion of resources, and constraints on commanders' freedom of action. Specific veterinary considerations that may point to BW attack include:

- Post-munitions disease. Disease following evidence of attack by munitions or aerosols. BA, however, may also be delivered covertly.

- The time-course of disease. The concurrent infection of large numbers of animals is rare in natural outbreaks. The sudden appearance of clinical signs in a high proportion of the population may therefore indicate delivery of BA. The dose, infectivity and lethality of BW agents may be higher than in natural infection, and they may cause sudden increases in animal mortality with few preceding signs of disease.

- The spatial distribution of disease. Multiple simultaneous outbreaks in separate locations are possible given animal movement during the latent period. However, this occurrence justifies suspicion of deliberate release. Alignment of cases with recent wind direction suggests airborne infection. If airborne infection is uncommon in natural infection this might suggest delivery by aerosol.

- Host range. The occurrence of a disease in a host species that is not normally affected in nature is suspicious. The simultaneous appearance of disease in several animal species is also highly unusual in natural infections.

- Geographical range. An illness highly unusual for the geographical area is suspicious, for example the occurrence of Venezuelan Equine Encephalitis outside the Americas. Likewise, the occurrence of disease in an area that lacks its natural reservoir or vector species.

- Predisposing conditions. Many diseases tend to occur in the presence of predisposing conditions, for example seasonality in vector abundance. The diagnosis of disease in the absence of these conditions may indicate atypical sources 
of infection. Conversely, unexpected observation of predisposing conditions, for example, unusual entomological patterns, may indicate attempts to artificially increase disease incidence.

- Route of infection. The route of delivery and infection may influence the development of clinical signs. For example, inhalational anthrax is normally rare in ruminants, while deliberate release in aerosol form may lead to an unusual predominance of respiratory signs.

- Heterogeneity of infection. Differences in the incidence of disease in groups of animals afforded different levels of protection, for example, animals housed or outdoors, may indicate different levels of exposure to a BA. However, be aware of possible confounding variation in the risk of natural infections.

- Background disease incidence. Recognition of disease in excess of expected levels requires knowledge of normal patterns of occurrence of animal disease in the Area of Responsibility (AOR). This forms an essential component of medical intelligence. The endemic presence of potential BW agents in a region does not exclude the possibility of deliberate release. Characterisation of patterns of infection, as well as aetiological diagnosis, is therefore critical to early recognition of outbreaks of disease and BW incidents.

\section{Aetiological Diagnosis}

Investigation of Incidents of Animal Disease or Mortality. Diagnosis of the agent causing disease is essential to the success of steps to prevent human exposure, and to the instigation of appropriate therapy in those personnel already exposed. For zoonoses, whether naturally-occurring or used in BW, this is likely to rely on examination and sampling of animals or carcasses for the agent, and for indirect indicators of infection such as pathological changes. The specialist nature of this task would require deployment of dedicated veterinary teams, which are then temporarily unavailable to respond to other incidents. Given limited resources in theatre, investigation of reported cases of animal disease or mortality should be selective, and take into account all available information concerning the perceived threat, patterns of disease reported by those at the scene, and expected occurrence of endemic disease or mortality in the AOR.

Detection and Diagnosis. Identification of disease or death in animals that could indicate a threat to man should be confirmed by aetiological diagnosis. This will usually involve clinical and/or pathological examination, and harvesting of samples for further analysis. This should be carried out with due regard to personal safety, biosecurity, selection of the most useful tests and samples, and appropriate carriage and analysis of samples.

\section{DIAGNOSIS AND SAMPLE COLLECTION}

\section{Diagnosis}

The accurate reporting of clinical findings may be critical in alerting other units to both the possibility and nature of a BW or CW attack. Unfortunately, attempts to reach a firm diagnosis on clinical grounds alone may not be productive. Rapid isolation and differential identification of the BA is then of prime importance for the local population and risk management. Emerging technology will be likely to provide provisional diagnostic capabilities locally. However, establishing a definitive diagnosis will often require specialised laboratory facilities. In some cases, clinical findings along with observed epidemiological patterns will be strong enough for presumptive diagnosis and enable instigation of appropriate force protection measures such as MedCM or ROM, which can be instigated while awaiting definitive diagnosis.

\section{Environmental Sampling}

Veterinary responsibilities will normally be limited to collection and submission of diagnostic materials from animals. Environmental sampling, however, is an important element in corroborating the occurrence of a BW attack and involves other agencies such as SIBCRA (Sampling and Identification of Biological Chemical and Radiological Agents) teams. Success or failure in providing a timely medical response will depend upon the rapidity and accuracy of the diagnostic effort, together with the passage of timely information from those organisations involved in environmental sampling. Close co-ordination and co-operation between NBC and Medical staffs will be vital to optimise sampling.

\section{Risks of Diagnostic Investigation}

The investigation of potentially zoonotic disease, whether deliberate release is suspected or not, poses risks to the investigator and to other personnel through:

- Infection at the time of investigation. Precautions considered adequate in the investigation of naturally occurring zoonotic disease may not provide good protection against BW. Agents used in BW may differ in their dispersion characteristics, infectivity and lethality when compared with their natural counterparts. Personal protection when investigating an unknown disease in animals in theatre should always err on the side of caution.

- Contamination at the site of investigation. Contamination of clothing or equipment, including sample containers, at the time of 
investigation may pose a health risk to the investigator and other personnel after returning from the site of disease occurrence, and could also precipitate disease and cycling of infection in susceptible animals subsequently encountered. BA especially formulated for delivery may be more liable to be dispersed in this way than their natural equivalents. Following BW attack, the environment as well as the animals under investigation may act as a source of contamination. Every effort should be made to avoid carrying contamination from the site of investigation.

\section{Safe Sampling, Specimen Handling and Shipment}

When conducting clinical and pathological investigation, and collecting and submitting samples, the following should be taken into account:

- Personal Protection. Personal protection should never be less than that normally recommended for clinical and post mortem examination. If there is reason to suspect infectious disease, and for all post mortem examinations where the cause of death is unknown, adequate dermal and respiratory protection should be used. The majority of infectious organisms will not cross normal clothing and intact skin; however, minor skin abrasions may be unnoticed, and surgical gloves should be worn. In this case, and in post mortem examination especially, particular attention should be paid to protecting the skin above the glove line. Some infections for example, $\mathrm{Q}$ fever, are readily transmitted by inhalation of a very small dose. Other BA are not transmitted by inhalation in natural infections, but delivery of BW as aerosolised organisms may increase the risk of infection by this route, from both the environment and affected animals. Residual chemical agent may also be inhaled from contaminated animals or carcasses. In every suspicious incident, therefore, Individual Protective Equipment (IPE) with respiratory protection is recommended. A facemask with eye protection will be adequate in most cases; if in doubt, a respirator should be worn. Anyone involved in examination and sampling should be appropriately trained and made aware of the risks.

- Contamination and Biosecurity. Infectious organisms or chemical agents may contaminate the operator and the equipment and containers used for sampling, and pose a risk to personnel in other locations. Ideally, outer protective clothing, gloves and packaging should be left at the site of investigation. Where this is not possible they should be treated as potentially infectious clinical waste and disposed of accordingly. IPE provides adequate skin protection where there is no possibility of exposure to BW contaminated liquids, such as blood and other body fluids as IPE will absorb such contaminants. Additionally, removal of IPE might not be possible if there is an ongoing environmental threat. In these cases, impervious outer protective clothing should be worn, and scrubbed with approved disinfectant (e.g. 10\% sodium hypochlorite) to remove contamination before leaving the site. The outside surface of specimen containers should be similarly disinfected, and samples placed into a second sealed container before leaving the site.

- Local Contamination. Every effort should be made to minimise the threat of increasing contamination of the local area with infectious organisms. This is of particular concern when escape of resistant forms into the environment can cause long term contamination and onward transmission, for example, anthrax spores. Samples should be taken as non-invasively as possible, and carcasses opened only where necessary for diagnosis. Post mortem examination should be conducted on an impervious surface where possible, for example plastic sheeting would suffice. If infectious disease is identified, provisions should be made for proper disposal of the carcass within a suitable time frame. In the meantime, the infected area should be clearly marked and access of both military and civilian persons restricted.

- Aseptic Technique. Aseptic technique should be used when collecting samples from animals or carcasses, as contaminants can confuse laboratory diagnosis.

- Samples Required. The samples taken should be based on signs observed and possible differential diagnoses. If possible, confirm sampling requirements with the destination laboratory. In the absence of specific advice, consult Table 1 for samples useful in the diagnosis of potential BA. The following are useful in most cases:

- Nasal swabs. Swabs should be placed in transport medium (bacterial and/or viral), and refrigerated at $4-8^{\circ} \mathrm{C}$. Viral swabs should be frozen if transport to the laboratory is likely to take longer than 2-3 days.

- Serum. Acute serum (at least $3 \mathrm{ml}$ for suspected infectious agents, and at least $20 \mathrm{ml}$ for suspected intoxications) should be collected as early as possible after onset of symptoms. Samples should also be obtained from exposed 
animals that are not yet symptomatic. Convalescent sera from survivors and non-affected individuals could be obtained 3-4 weeks later if necessary to confirm the diagnosis. Serum may be refrigerated or frozen for transport.

- Blood. Blood culture with routine media will readily detect many bacterial agents, although specialised media may be required for some. Both aerobic and anaerobic cultures should be obtained routinely. Sodium citrate is the anticoagulant of choice for blood intended for culture, and samples should also be refrigerated at $4-8^{\circ} \mathrm{C}$. Blood should not be frozen.

- Tissues. Tissue samples obtained at post mortem should be collected in multiple aliquots: minimally, one (2550 grams) to freeze for microbial or toxin analysis and one in 10\% formalin for histopathology. Where possible, additional specimens for specialised procedures such as immunofluorescence or polymerase chain reaction (PCR) studies should be obtained. Organs sampled should generally include lung, local and mediastinal lymph nodes, spleen, and liver. Obvious lesions and adjacent normal tissue should be taken from affected areas of any organ. Post mortem blood (up to $20 \mathrm{ml}$ ) should be obtained. Fluid for culture and impression smears may be taken from involved lymph nodes, sputum, pleural fluid, cerebrospinal fluid (CSF), spleen and urine. Appropriate swabs may also be taken from lesions. Small animals (e.g. small rodents) may be placed whole in a suitable sealed container (e.g. 'biopot' - as used by SIBCRA), refrigerated and sent directly for analysis. Transport of carcasses within theatre without strict biosecurity precautions is ill advised.

- Specimen Labelling. Samples should be placed in suitable sealed containers and clearly labelled with individual identification, species, type of specimen, date of collection, and geographical origin. A brief description of the illness and/or gross post mortem findings should be included, along with the date and time of collection and - where known estimated dates of exposure, onset of clinical signs, course of disease and death. Additionally, a description of the epidemiology of the disease (both human and animal) within the locality is required.

- Sample Inner Packaging. Serum and other samples should be placed in securely sealed vials or containers. If possible, each sample should be individually placed in a second plastic vial or zip-top bag to prevent leakage. All specimens should be contained in a metal shipping can or other secondary container with sufficient absorbent material packed to prevent leakage outside the container; the SIBCRA process will provide this outer packaging. Samples for microbial or toxin analysis should be kept refrigerated $\left(4-8^{\circ} \mathrm{C}\right)$. If analysis is not to take place within 72 hours, samples should be frozen, with the exception of formalin-fixed material that can be stored at ambient temperature but must not be frozen.

- Shipping to Laboratory. Shipping of samples to the home base will be organised by the National Component Command (NCC) utilising the SIBCRA process. The destination laboratory will be informed by the NCC of the shipment of samples, to enable them to make appropriate arrangements for receipt. Medical laboratories in theatre and elsewhere may not be willing to accept samples from animals, especially if containing potential zoonoses. Arrangements for laboratory analysis should, therefore, be made by the NCC prior to shipping samples. Diagnostic facilities available in theatre might include routine staining, culture, and in some cases PCR. Duplicate samples may be analysed in parallel: in theatre for rapid preliminary identification, and simultaneously out of theatre in a specialised laboratory for definitive diagnosis.

- Chain of Custody. Records should be kept of the origin, route and destination of samples. Where BW is suspected, custody of samples should be formally handed over and recorded at each stage. Clinical and operational data should also be included for all samples. These requirements must be strongly and clearly delineated since evidence may well be politically or militarily disputed. The need to satisfy international law and prove the chain of evidence will be paramount after a BW attack. Handling and submission of samples after a suspected BW attack should be done in consultation with SIBCRA and other specialist agencies.

- Informing Command. When investigating a suspect incident the NCC must be informed as early as possible in the process. This will enable early activation of the SIBCRA process if appropriate and allow consideration to be given to placing Specialist Courier Teams (SCT) on standby, to assist rapid delivery of samples. 


\section{IDENTIFICATION OF SPECIFIC BW AGENTS}

Identification Methods

Methods of identification of BW agents include:

- Isolation of the aetiological agent by culture (possible in one to two days for some BA, but slow or routinely unsuccessful in others).

- Detection of toxin by animal inoculation or in vitro methods.
- Antibody detection (specific immunoglobulin $M(\operatorname{IgM})$ may appear within 3 days).

- Antigen detection via enzyme immunoassay or other sensitive assay methods (eg immunofluorescence).

- Genome detection employing DNA probes or Polymerase Chain Reaction (PCR).

- Detection of metabolic or degradation products of the infectious agent or toxin in clinical specimens.

- (Electron) Microscopy.

\begin{tabular}{|c|c|c|c|c|c|c|c|}
\hline AGENT & $\begin{array}{l}\text { FACE OR } \\
\text { NASAL } \\
\text { SWAB }\end{array}$ & $\begin{array}{l}\text { BLOOD } \\
\text { CULTURE }\end{array}$ & SMEAR & \begin{tabular}{|c|} 
ACUTE/ \\
CONVALESCENT \\
SERA
\end{tabular} & STOOL & URINE & OTHER \\
\hline Anthrax & + & + & Peripheral blood & + & + & - & Spleen, mediastinal lymph nodes for culture \\
\hline Brucella & + & + & - & + & - & - & $\begin{array}{l}\text { Aborted foetal material (stomach, lungs); } \\
\text { placental swabs or tissue for culture; milk }\end{array}$ \\
\hline Glanders & + & - & Lesions & + & - & - & Lymph node aspirates or lesions for culture \\
\hline Melioidosis & + & - & - & + & - & - & $\begin{array}{l}\text { Lymph node aspirates or lesions for culture; } \\
\text { spleen }\end{array}$ \\
\hline Plague & + & + & Sputum (lab. only) & + & - & - & Bubo aspirate/lymph nodes, CSF, sputum \\
\hline Q-Fever & + & + & - & + & - & - & Aborted material / vaginal swab, milk \\
\hline Rift Valley Fever & + & + & - & + & + & - & Liver, lymph nodes, spleen for culture \\
\hline Tularaemia & + & + & + & + & - & - & Liver lesions, lymph nodes, spleen for culture \\
\hline $\mathrm{CCHF}$ & - & - & - & + & - & - & \\
\hline VEE & + & + & - & + & - & - & CSF, brain \\
\hline Botulism & + & - & - & + & - & - & Liver, brain \\
\hline Clostridial Toxins & + & - & Wound tissues & + & + & - & - \\
\hline SEB Toxin & + & - & - & + & + & + & Lung, kidney \\
\hline Ricin Toxin & + & - & - & + & + & + & Spleen, lung, kidney \\
\hline Chemical Agents & + & - & Skin & + & - & + & Lung, liver, kidney, CSF \\
\hline
\end{tabular}

Table 1. Specimens for laboratory dianosis for potential $B W$ agents in animals. 